\title{
Human embryonic stem cells from aneuploid blastocysts identified by pre-implantation genetic screening
}

\author{
Kavita Narwani • Juan-Carlos Biancotti • \\ Tamar Golan-Lev • Nicole Buehler • David Hill • \\ Sagiv Shifman • Nissim Benvenisty $•$ Neta Lavon
}

Received: 31 March 2009/Accepted: 19 January 2010 /Published online: 12 March 2010 / Editor: P. Andrews

(C) The Author(s) 2010. This article is published with open access at Springerlink.com

\begin{abstract}
Human embryonic stem cells are derived from the inner cell mass of pre-implantation embryos. The cells have unlimited proliferation potential and capacity to differentiate into the cells of the three germ layers. Human embryonic stem cells are used to study human embryogenesis and disease modeling and may in the future serve as cells for cell therapy and drug screening. Human embryonic stem cells are usually isolated from surplus normal frozen embryos and were suggested to be isolated from diseased embryos detected by pre-implantation genetic diagnosis. Here we report the isolation of 12 human embryonic stem cell lines and their thorough characterization. The lines were derived from embryos detected to have aneuploidy by pre-implantation genetic screening. Karyotype analysis of these cell lines showed that they are euploid, having 46 chromosomes. Our interpretation is that the euploid cells originated from mosaic embryos, and in vitro selection favored the euploid cells. The undifferentiated cells exhibited long-term proliferation and expressed markers typical for embryonic stem cells such as OCT4, NANOG, and TRA-160. The cells manifested pluripotent differentiation both in vivo and in vitro. To further characterize the different lines, we have
\end{abstract}

K. Narwani and JC. Biancotti contributed equally to this work.

K. Narwani $\cdot$ J.-C. Biancotti $\cdot$ N. Benvenisty $\cdot$ N. Lavon

The International Stem Cell Research Institute,

Cedars-Sinai Medical Center,

Los Angeles, CA, USA

T. Golan-Lev $\cdot$ S. Shifman $\cdot$ N. Benvenisty $\cdot$ N. Lavon $(\varangle)$

Department of Genetics, The Institute of Life Sciences,

The Hebrew University,

Jerusalem, Israel

e-mail: neta.lavon@mail.huji.ac.il

N. Buehler $\cdot$ D. Hill

ART Reproductive Center,

Beverly Hills, CA, USA analyzed their ethnic origin and the family relatedness among them. The above results led us to conclude that the aneuploid mosaic embryos that are destined to be discarded can serve as source for normal euploid human embryonic stem cell lines. These lines represent various ethnic groups; more lines are needed to represent all populations.

Keywords Human embryonic stem cells · Derivation . Aneuploidy Pre-implantation genetic screening .

Cell repository

\section{Introduction}

Human embryonic stem cells (HESCs) are derived from the inner cell mass (ICM) of pre-implantation embryos (Thomson et al. 1998). HESCs have the unique properties of unlimited proliferation potential and capacity to differentiate into the cells comprising the entire human body (Itskovitz-Eldor et al. 2000; Schuldiner et al. 2000). The cells may serve as a source of cells for cell therapy and drug screening. These cells are also being used for the study of human embryogenesis and disease modeling.

HESCs are isolated from surplus embryos of in vitro fertilization (IVF) treatments (Thomson et al. 1998; Reubinoff et al. 2000). One common source of surplus normal embryos is couples that have undergone IVF treatments and have finished their family planning and have donated their frozen blastocysts for research. Other sources of embryos for research are poor-quality embryos, morula-stage embryos, and single blastomeres (Mitalipova et al. 2003; Strelchenko et al. 2004; Klimanskaya et al. 2007). Embryos carrying genetic mutations detected by pre-implantation genetic diagnosis (PGD) or aneuploid embryos carrying abnormal chromosomal number diagnosed by pre-implantation genetic 
screening (PGS) may also serve as sources for HESC lines. The embryos going through PGD/PGS are commonly biopsied at the eight-cell stage, corresponding to 3-d old embryos. A hole is drilled in the zona pellucida (ZP), and one or two of the blastomeres are removed for genetic analysis. By the time the embryo reaches its blastocyst stage (5-d old embryo), the healthy embryos are identified and separated, and the abnormal embryos are either discarded or used for research purposes. In PGD, the specific mutation that is inherited in the family is usually analyzed by PCR (Ao et al. 1996). Among the genetic diseases that HESC lines were derived from are Duchenne muscular dystrophy, Fanconi anemia, and Fragile-X syndrome (Pickering et al. 2005; Verlinsky et al. 2005; Eiges et al. 2007). PGS is widely applied to identify chromosomal aneuploidies during IVF, especially in cases of advanced maternal age. In PGS, blastomeres are analyzed using single-cell fluorescent in situ hybridization, which allows the analysis of chromosomes in interphase cells using multicolor fluorescent probes for five to 12 chromosomes (Griffin et al. 1991). Embryos that are detected as carrying chromosomal aneuploidies are destined to be discarded unless donated for research. The widespread use of PGS in some IVF clinics has created a new source of surplus blastocyst-stage embryos that are available for the establishment of HESC lines (Lavon et al. 2008).

We have previously shown that we can derive euploid HESC lines, with normal karyotype, from blastocyst-stage embryos that were diagnosed by PGS as aneuploid (Lavon et al. 2008). Using polymorphic markers, we have shown that the euploidy was not achieved through chromosomal self correction. Instead, we have suggested that the euploid HESC lines originated from mosaic embryos consisting of aneuploid and euploid cells, and in vitro selection occurred to favor euploid cells (Lavon et al. 2008). These results led us to conclude that the aneuploid mosaic embryos that are destined to be discarded can serve as an alternative source for normal euploid HESC lines.

In this report, we summarize the data that we have collected from the derivation of a dozen HESC lines, carrying euploid karyotypes. The HESC lines exhibited morphology and expressed markers typical of HESCs and also demonstrated a capacity for long-term proliferation. The derived HESC lines manifested pluripotent differentiation both in vivo and in vitro. To further characterize the HESC lines, we have analyzed their ethnic origin and the family relatedness among them.

\section{Materials and Methods}

Derivation of HESCs from blastocysts after PGS. Isolation of HESCs from blastocyst-stage embryos was performed under the approval of the Stem Cell Research Oversight Committee at
Cedars-Sinai Medical Center, according to protocol \#9647: "Establishment of HESCs from Genetically Abnormal and Spare IVF-Derived Embryos." Blastocyst-stage embryos were donated according to the informed consent under the approved protocol. Blastocysts that did not completely hatch from the ZP were either left over night to spontaneously hatch or were assisted mechanically to release the blastocyst from the ZP (Lavon et al. 2008). Thus, we have used 6- to 7-d-old naked blastocysts for isolating the ICM. In most cases, the trophectoderm (TE) was removed by immunosurgery (IS), and a minority required mechanical dissection in order to remove the TE layer from the ICM (Eiges et al. 2007). Using a stripper, a naked blastocyst was transferred to a drop of antihuman serum (Sigma, St. Louis, MO) diluted 1:5 with G2V3 blastocyst medium supplemented with human serum albumin (Vitrolife, Englewood, CO) and incubated for $30 \mathrm{~min}$ at $37^{\circ} \mathrm{C}$ in a $5 \% \mathrm{CO}_{2}$ incubator. Then the blastocyst was washed three times with the blastocyst medium, and the blastocyst was transferred to a drop of guinea pig complement (Sigma) diluted 1:5 with blastocyst medium for $30 \mathrm{~min}$ at $37^{\circ} \mathrm{C}$ in a $5 \% \mathrm{CO}_{2}$ incubator. The blastocyst was washed again three times with the blastocyst medium, and blebbing of the TE was apparent. After the TE blebbing, if the TE was not completely removed from the ICM, it was removed by passing the blastocyst through a narrow pipette. The ICM was placed on mouse embryonic fibroblasts (MEF) as a feeder layer with HESC medium (Itskovitz-Eldor et al. 2000) supplemented with $30 \mathrm{ng} / \mathrm{ml}$ of basic fibroblast growth factor (bFGF). The medium was changed every $2 \mathrm{~d}$, and after 5-12 d, when the colony was around $300 \mu \mathrm{m}$ in diameter, it was cut (using a filter-isolated mouth pipette) into two to three parts and transferred to new plates. The new colonies were transferred every 5-9 d, using mouth pipette, for the first few wk. At passages $4-8$, as the cell line is more established, we used either trypsin/EDTA or collagenase type IV for transferring the cells (Itskovitz-Eldor et al. 2000). Once the cell line was established, we lowered the bFGF concentration to $5 \mathrm{ng} / \mathrm{ml}$. The CSES cell lines can be obtained from Cedars-Sinai Medical Center for research purposes.

In vitro and in vivo differentiation of HESCs. Embryoid bodies (EBs) were created as described previously (ItskovitzEldor et al. 2000). EBs were generated by aggregation of the HESCs for $10 \mathrm{~d}$. For teratoma formation, $5 \times 10^{6}$ HESCs were injected under the kidney capsule of 6-8-wk-old nude mice (Robertson 1987). Each cell line was injected into two mice. One month later, the mice were euthanized, and the teratomas were removed. Part of the tissue was fixed by $10 \%$ buffered formalin phosphate (Sigma), and RNA was extracted from the rest of the tissue as detailed below. The fixed tissues were embedded in paraffin, and 4- $\mu \mathrm{m}$ sections were stained by hematoxylin and eosin. Care of animals was in accordance with institutional guidelines as approved by the Cedars-Sinai 
Medical Center Institutional Animal Care and Use Committee, according to protocol \#2182.

Staining for stem cell markers. For immunostaining, HESCs were washed three times with PBS and fixed onto the plate with $10 \%$ buffered formalin phosphate (Sigma). As primary antibodies, we used mouse IgG anti-human OCT-3/4, mouse IgM anti-human TRA-1-60 (Santa Cruz Biotechnology Inc, Santa Cruz, CA) and rabbit anti-NANOG (Abcam Inc, Cambridge, MA). As secondary antibodies, we used goat anti-mouse IgG PE, goat anti-mouse IgM FITC, and donkey anti-rabbit FITC (Santa Cruz Biotechnology Inc). The primary antibody was incubated with the cells over night at $4^{\circ} \mathrm{C}$. The next morning the cells were washed twice with PBS and were incubated with the secondary antibody for $1 \mathrm{~h}$, at room temperature. The cells were washed, and nucleus staining was performed by incubation with $1 \mu \mathrm{g} / \mathrm{ml}$ of bisBenzimide, Hoechst no 33258 (Sigma) for $10 \mathrm{~min}$.

To analyze the alkaline phosphatase activity in the cells, HESCs grown in a 12-well plate were fixed and labeled with the alkaline phosphatase kit 86R-1KT (Sigma).

Figure 1. Undifferentiated HESCs of the CSES lines express alkaline phosphatase. Elevated expression of the enzyme alkaline phosphatase is associated with undifferentiated pluripotent stem cells. The CSES lines exhibit typical morphology of defined HESC colonies of small undifferentiated cells on a MEF feeder layer, and the colonies are positively stained for alkaline phosphatase (red). Scale bar is $100 \mu \mathrm{m}$.
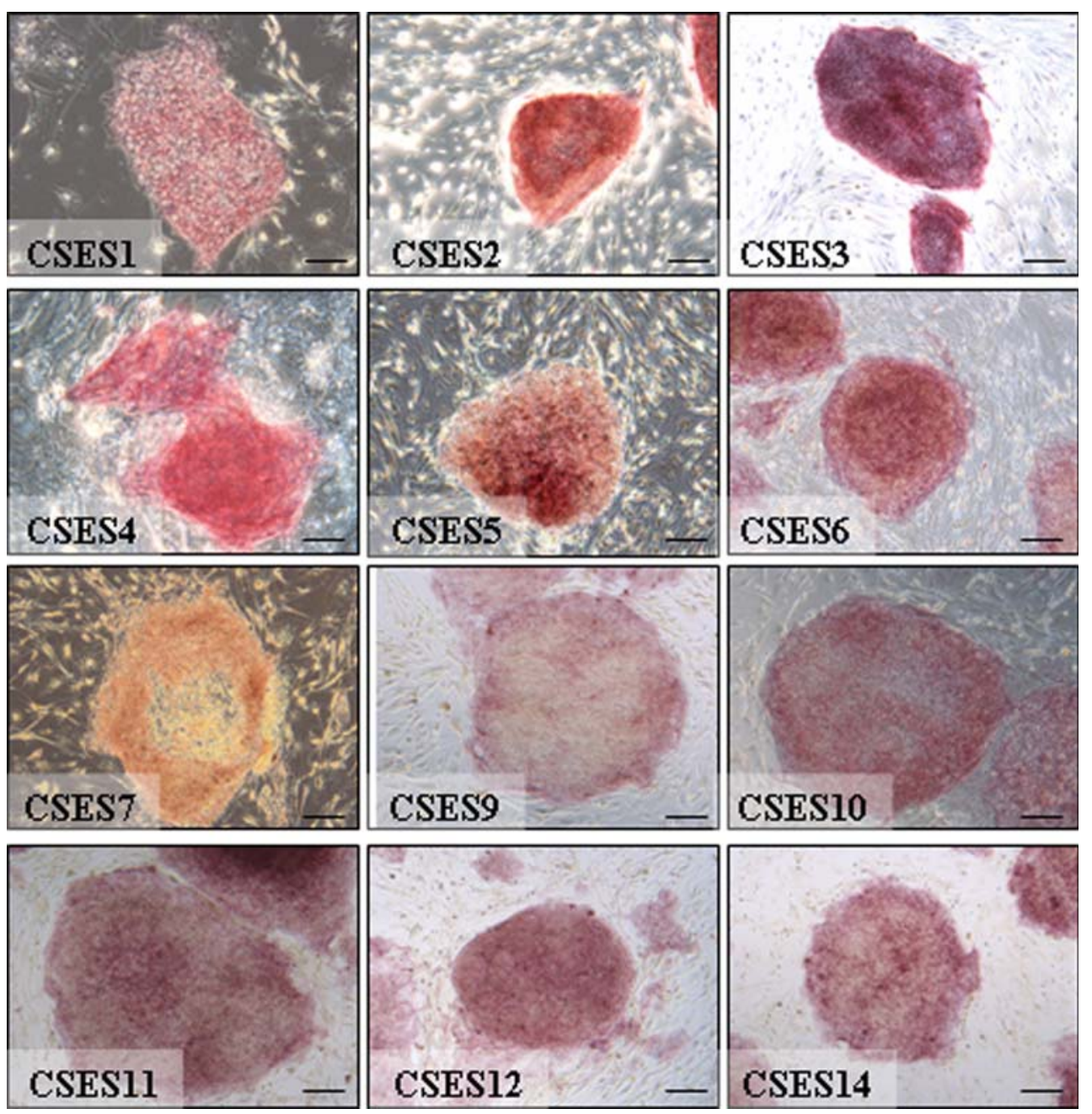
reported as DeltaCt values. For each individual gene, the number of amplification cycles for the fluorescent reporter signal to reach a common threshold value $(\mathrm{Ct})$ was estimated, and then normalized by subtracting the $\mathrm{Ct}$ value obtained for the same sample for a positive control transcript (beta actin), to give DeltaCt value.

Karyotype analysis. A 10-cm plate of HESCs in log growth phase was used from each cell line. The night before the procedure, the cells were fed with fresh $10 \mathrm{ml}$ HESC media. Next morning, KaryoMAX colcemid* (Invitrogen, Carlsbad, CA) was added directly to the plate, to give a final concentration of $100 \mathrm{ng} / \mathrm{ml}$, and the cells were incubated for $30 \mathrm{~min}$ at $37^{\circ} \mathrm{C}$ in a $5 \% \mathrm{CO}_{2}$ incubator. Then cells were trypsinized, treated with hypotonic solution, and fixed. Metaphases were spread on microscope slides, and by using standard $\mathrm{G}$ banding technique, the chromosomes were classified according to the International System for Human Cytogenetic Nomenclature (ISCN). At least 20 metaphases were analyzed per cell line, and the final karyotype was stated if at least $80 \%$ of the metaphases had it.
Analysis of the main ancestry components of the HESC lines. DNA samples were extracted from the dozen HESC lines, and their SNP profile was stated by Genechip Human Mapping $500 \mathrm{~K}$ Array Set (Afffymetrix, Santa Clara, CA). The main ancestry components of the different HESC lines was characterized based on genome-wide average proportion of alleles shared identical by state (IBS) between any two samples. IBS was calculated using the genotypes of 50,000 autosomal SNPs, randomly chosen from the NspI Affymetrix GeneChip Mapping Array. We analyzed the cell lines together with samples with known ancestry (HapMap samples from northern and western Europe, Japan, China and Africa). To visualize the population genetic structure, we plotted the results of a multidimensional scaling (MDS) analysis. The MDS algorithm was used with the IBS matrix of pairwise similarities, to assign a location to each sample in twodimensional space. Identical by descent (IBD) analysis was used to identify relatedness between samples. Calculations of IBS and IBD and the MDS analysis were performed using PLINK (Purcell et al. 2007).
Figure 2. Undifferentiated HESCs of the CSES lines express OCT4/NANOG. Representative HESC colony from each of the HESC lines is positively stained for transcription factor serving as markers of undifferentiated HESCs: POU5F1(OCT4) and NANOG. These genes are closely associated with the pluripotent state of HESCs and are strongly downregulated upon their differentiation. Red or green labeling for OCT4 (CSES1-7, 9 , and 14) and green labeling for NANOG (CSES10 and 11). Hoechst stain of nuclei of HESCs and MEFs is in blue. Scale bar is $100 \mu \mathrm{m}$.
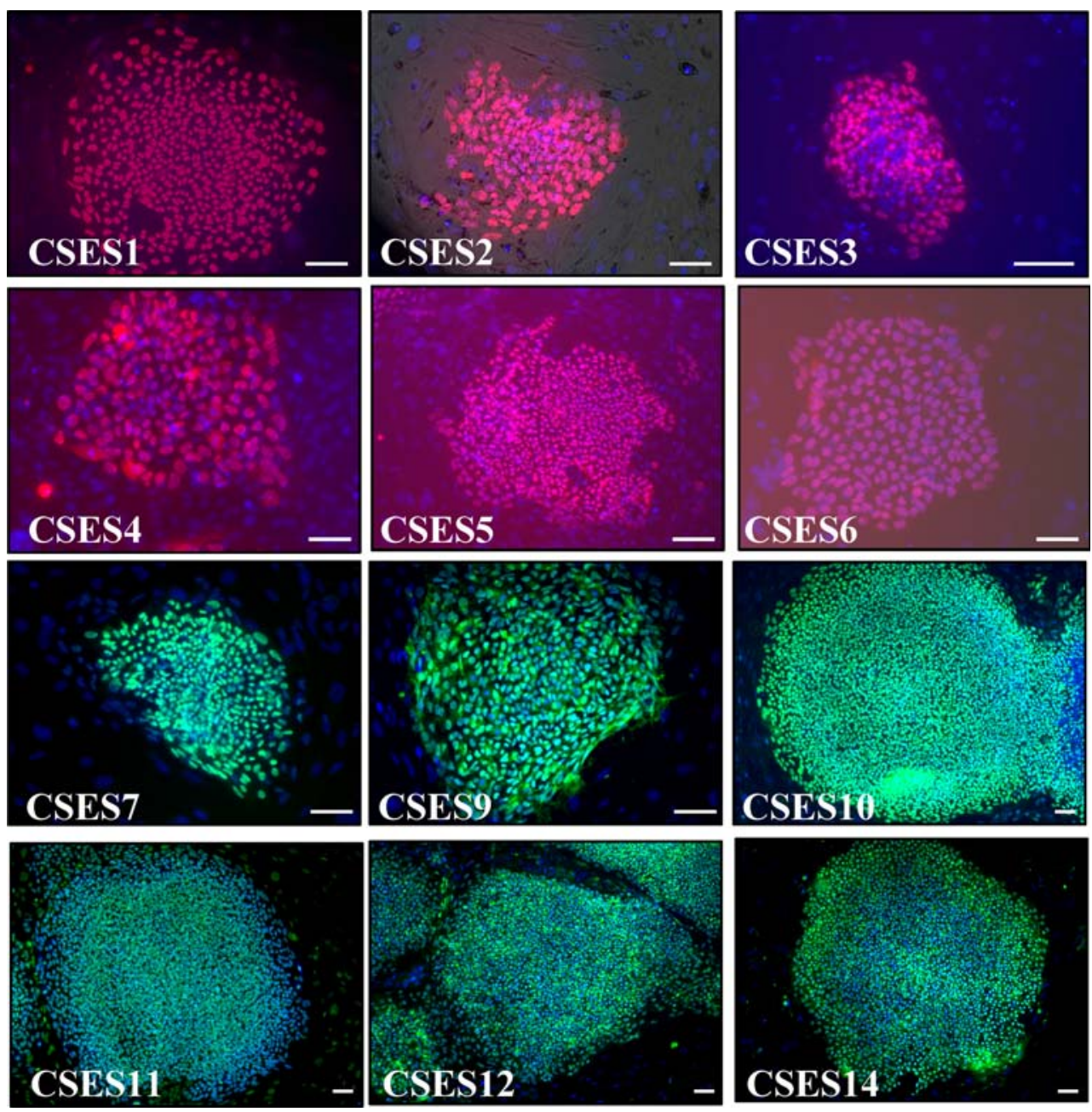
Table 1. FACS analysis of undifferentiated ES cells

\begin{tabular}{|c|c|c|c|c|c|c|c|c|c|c|c|c|}
\hline $\begin{array}{l}\text { FACS } \\
\%\end{array}$ & $\begin{array}{l}\text { CSES1 } \\
\text { + }\end{array}$ & $\begin{array}{l}\text { CSES2 } \\
\text { ㅇ }\end{array}$ & $\begin{array}{l}\text { CSES3 } \\
+\end{array}$ & 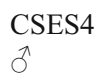 & $\begin{array}{l}\text { CSES5 } \\
+9\end{array}$ & $\begin{array}{l}\text { CSES6 } \\
\text { o }\end{array}$ & $\begin{array}{l}\text { CSES7 } \\
+\underset{+}{ }\end{array}$ & $\begin{array}{l}\text { CSES9 } \\
0\end{array}$ & $\begin{array}{l}\text { CSES10 } \\
q\end{array}$ & $\begin{array}{l}\text { CSES11 } \\
q\end{array}$ & 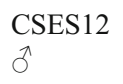 & $\begin{array}{l}\text { CSES14 } \\
\text { ㅇ }\end{array}$ \\
\hline SSEA4 & 85.4 & 82.6 & 86 & 79.4 & 86.6 & 84.35 & 83.6 & 84 & 78 & 91.7 & 93.4 & 95.8 \\
\hline TRA-1-60 & 78.2 & 78.4 & 83.8 & 82.3 & 90.6 & 88.68 & 86.6 & 87 & 84 & 91.1 & 92.0 & 94.4 \\
\hline
\end{tabular}

\section{Results}

In this report, we summarize the data that we have collected from the derivation of a dozen HESC lines, carrying euploid karyotypes. We started with fresh PGS-defined aneuploid blastocyst-stage embryos carrying various chromosomal abnormalities as trisomy of chromosomes 14, 15, or 18 and monosomies of chromosomes 14, 16, 18, or 21. As shown before, most of the embryos are mosaic, and the in vitro culture conditions during the derivation of the HESCs causes a strong selection for the euploid normal cells (Lavon et al. 2008). The HESC lines derived from these aneuploid embryos were karyotyped and found to have euploid karyotype: nine female $(46, \mathrm{XX})$ and three male $(46, X Y)$. CSES14 was exceptional due to the fact that on the top of the 46 chromosomes, it had in most of the cells a translocation of segment from chromosome 17 to chromosome 7 having a karyotype of $46 \mathrm{XX}, \mathrm{t}(7 ; 17)$.

In order to define the new cell lines as HESCs, we needed to demonstrate that the cells express typical markers of undifferentiated cells, have a self-renewal capacity, and have the pluripotent ability to differentiate into derivatives of the three embryonic germ layers. Elevated expression of the enzyme alkaline phosphatase is associated with undifferentiated pluripotent stem cells. In Fig. 1, we show that the dozen HESC lines have alkaline phosphatase activity. The HESCs show typical morphology of HESC colonies grown on MEFs, and only the HESC colonies are positively
Figure 3. Undifferentiated HESCs of the CSES lines express TRA-1-60. The keratan sulfate antigen TRA-1-60 is highly expressed in undifferentiated HESCs. The dozen HESC lines were analyzed by FACS, and the cultures grown on MEFs had $78-95 \%$ positive cells (shown in Table 1). Presented are HESC colonies from each of the dozen HESC lines stained for TRA-1-60. Scale bar is $100 \mu \mathrm{m}$.
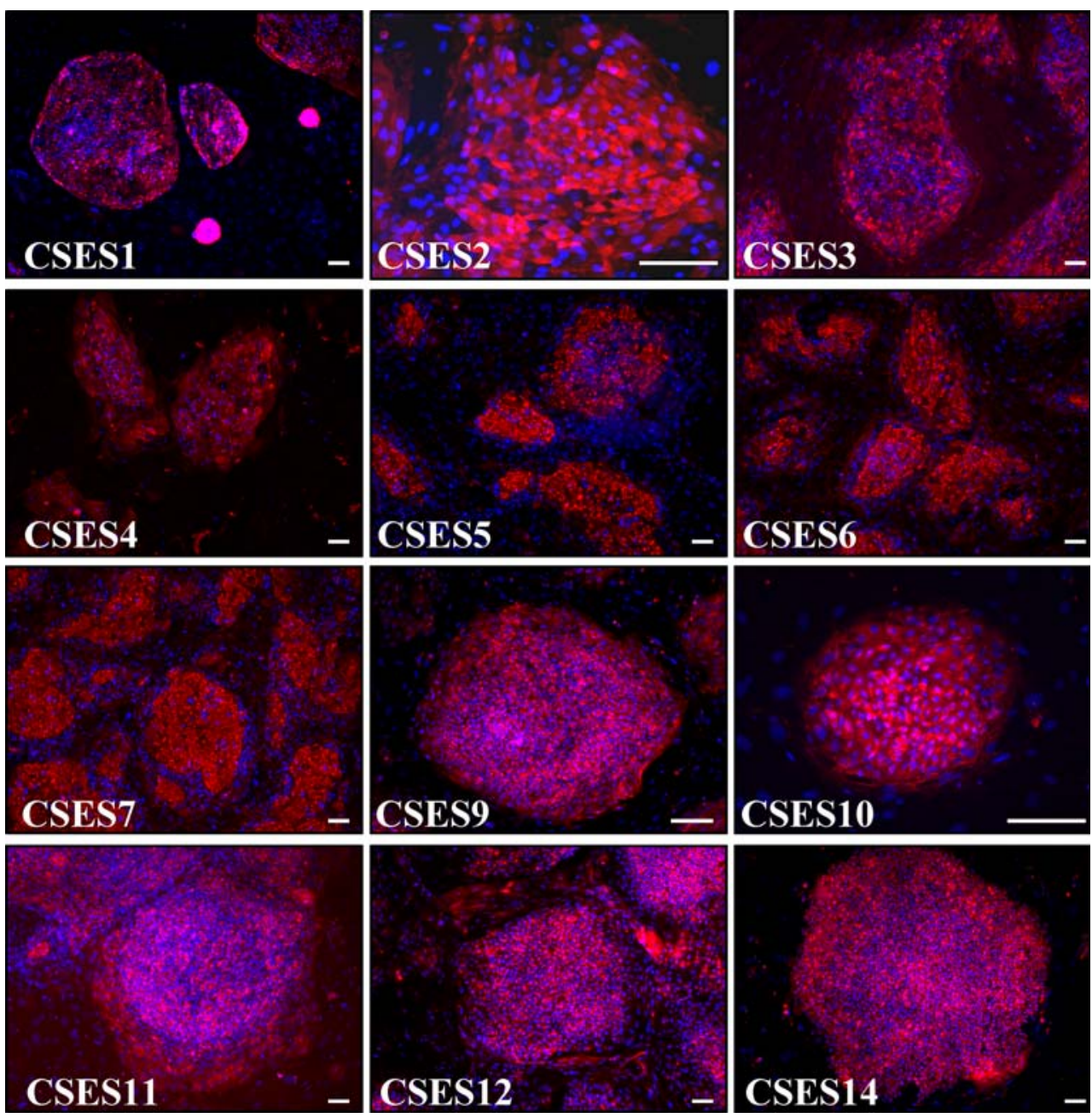
stained. POU5F1(OCT4) and NANOG are regarded as archetypal HESC markers (Adewumi et al. 2007). These genes had been previously proposed as markers of undifferentiated HESCs. They are closely associated with the pluripotent state of HESCs and strongly downregulated upon their differentiation. In Fig. 2, we show that the HESC lines express these archetypal HESC markers, as revealed by immunostaining with anti-OCT4 (CSES1-7, 9, and 14) and anti-NANOG (CSES10 and 11) antibodies. By FACS, we examined the expression of cell-surface antigens that are widely used as markers of HESCs such as the glycolipid SSEA4 and the keratan sulfate TRA-1-60 antigens. Table 1 details the results of the FACS analysis of the HESCs grown on MEF with antibodies against TRA-1-60 and SSEA4; 78$96 \%$ of the cells are positive for these HESCs surface markers. The cells remained over $80 \%$ SSEA4 and TRA-160 positive in low (P5-P15) and high passages (P25+) (data not shown). We further tested the expression of the surface marker, TRA-1-60, by immunostaining. In Fig. 3, we show that the HESC colonies are positively stained for TRA-1-60 in contrast to the MEF feeder layer. To examine cell pluripotency, we tested their differentiation potential in vitro and in vivo. In Fig. 4, we demonstrate the differentiation potential of our different HESC lines upon in vivo differentiation. All the HESC lines generated teratomas in mice as shown by the sections of the teratomas stained with hematoxylin and eosin. The teratomas are comprised of a variety of cell structures and cell types. Among the structures seen in the various teratomas are neural rosettes, gut-like structures, fat tissue, and cartilage foci. In vitro, we have created EBs from all the cell lines and tested their expression profile upon differentiation. The differentiated cells showed elevated expression levels of the genes serving as markers for differentiation into ectoderm, mesoderm, endoderm, and trophectoderm. In Fig. 5, we demonstrate the expression profile of a representative cell line (CSES11) upon in vitro differentiation. We observe upregulation in the expression of these marker genes upon in vitro differentiation of the cells. The average expression of each gene presented is higher in the EBs compared to the undifferentiated cells. To further characterize our HESCs, for each of the HESC lines, we have analyzed its global SNP profile. This data allowed us to
Figure 4. Upon in vivo differentiation, the CSES lines generate teratomas. Upon in vivo differentiation into teratomas, the CSES cell lines differentiate into a variety of structures and comprised of heterogeneous cell types; shown are 4- $\mu \mathrm{m}$ sections of teratomas stained with hematoxylin and eosin. In all cell lines, we have seen presentation of structures from the three embryonic germ layers. Shown are variety of structures and cell types including neural rosettes, gut-like structures, cartilage, connective tissue, adipose, and muscle cells. Scale bar is $100 \mu \mathrm{m}$.

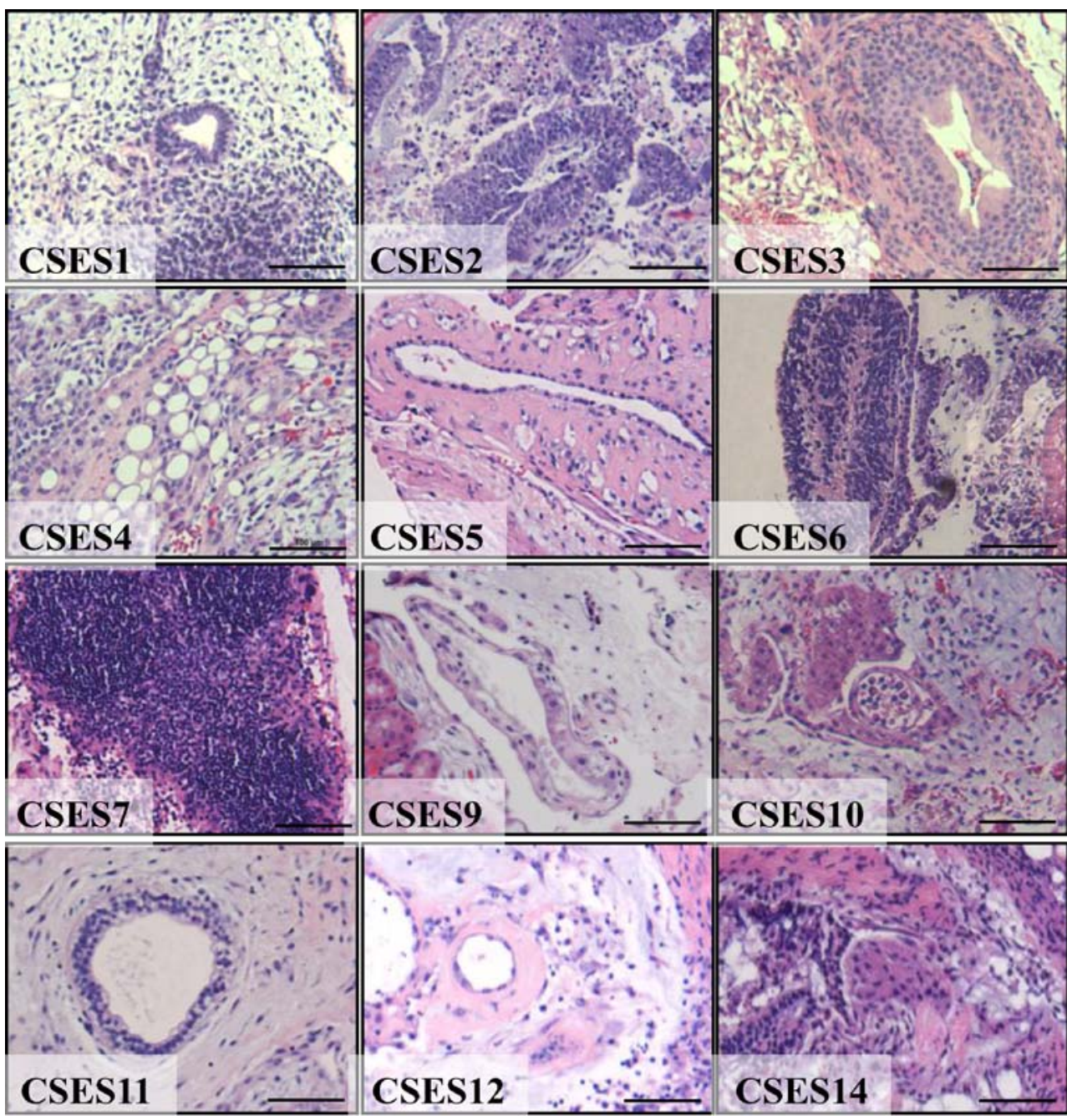




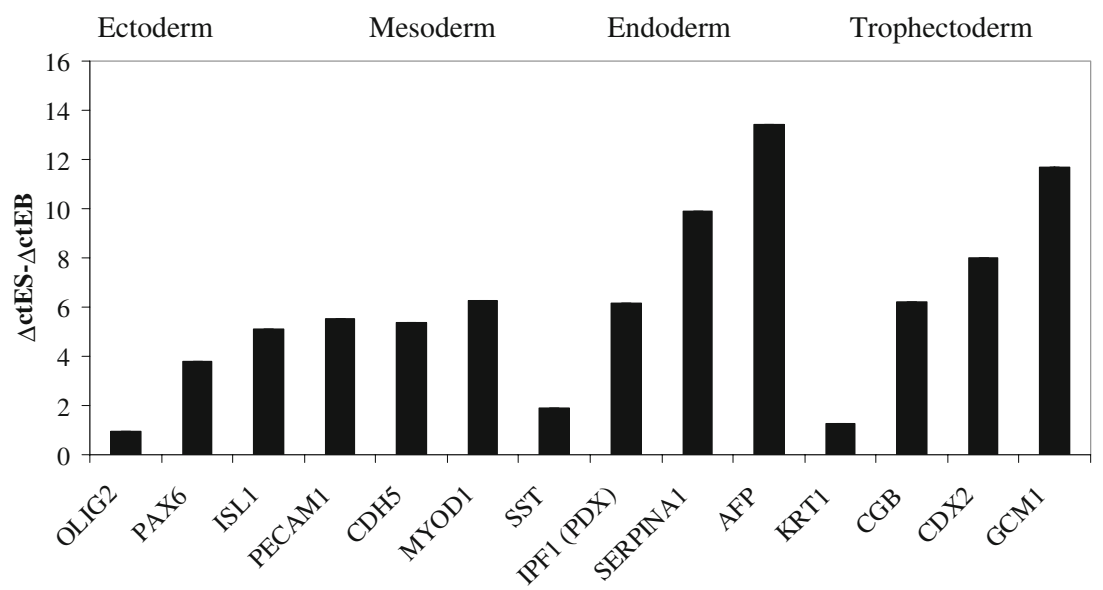

Figure 5. Upon in vitro differentiation, the CSES lines generate EBs expressing the differentiation markers for ectoderm, mesoderm, endoderm, and trophectoderm. Markers representing each of the germ layers are presented in the graph; ectoderm (OLIG2, PAX6, and ISL1), mesoderm (PECAM1, CDH5, and MYOD1), endoderm (SST, PDX1, SERPINA1, and AFP), and trophectoderm (KRT1, CGB,

have the unique footprint of each HESC line. Moreover, the data allowed us to explore the ethnic origin (the main ancestry components) of the different HESC lines and possible family relatedness among them. Figure 6 shows the results of a multidimensional scaling analysis. Most lines (eight out of 12) fall within (CSES2, CSES3, CSES9, and CSES10) or very near (CSES1, CSES4, CSES5, CSES6, and CSES14) the northern and western European cluster. The cell lines falling near the western European cluster could be of Eastern European or Ashkenazi Jewish ancestry (Price et al. 2008). The three lines falling far from the European cluster include CSES12 that falls within the Chinese cluster (Fig. 6, inset), and CSES7 and CSES11 that are spaced between the European and the Asian clusters, suggesting a possible admixture effect. IBD analysis identified high IBD level between CSES5 and CSES6 (0.47) and between CSES9 and CSES10 (0.46), suggesting that they are derived from the same parents ("full sibs"). No other family relatedness was found between the other cell lines.

\section{Discussion}

We have derived 12 new HESC lines from PGS-biopsied embryos and have shown that they express markers of undifferentiated cells, have a self-renewal capacity, and have the pluripotent ability to differentiate into derivatives of the three embryonic germ layers (Figs. 1, 2, 3, 4, and 5 and Table 1). To further characterize the HESCs, we stated the main ancestry components of the different HESC lines and possible family relatedness among them (Fig. 6).

The embryos that we used for the derivation of new HESC lines were reported to have chromosomal aberrations by PGS.
GCM1, and CDX2). The results presented are from the expression data of CSES11. Each bar represents the average induction in the expression of each gene upon differentiation into EBs. An increase in the delta ct ES-delta ctEB value of 1 denotes a twofold increase in the mRNA levels of this gene upon differentiation to EBs.

These embryos were destined to be discarded. From these embryos, we have derived HESC lines that were found to carry a normal euploid karyotype. These HESC lines were characterized as pluripotent cells having self-renewal capacity,

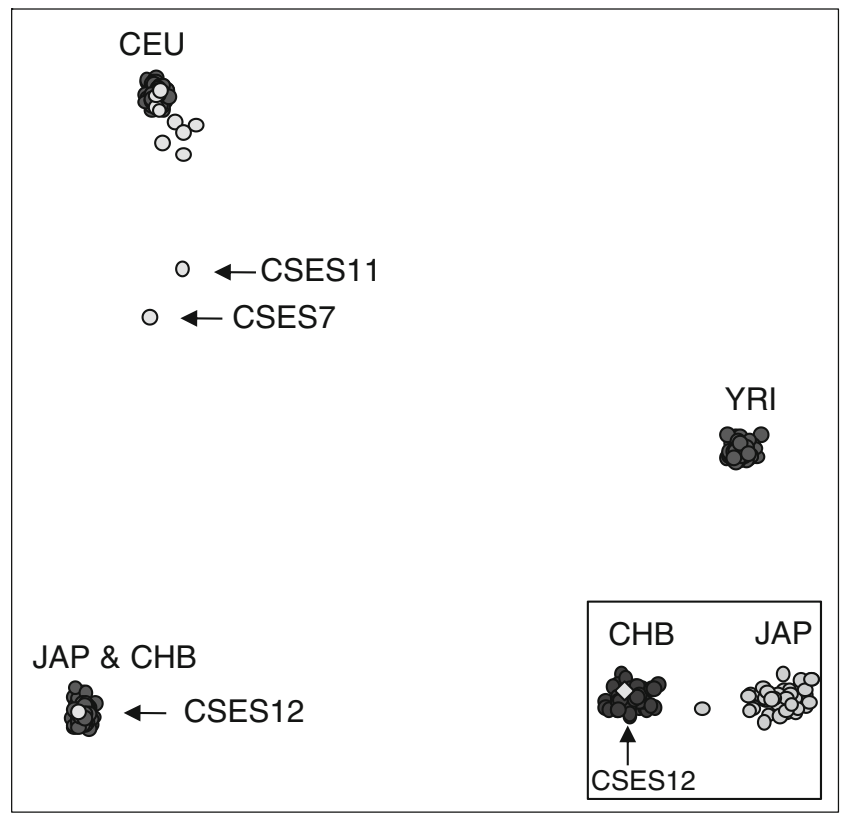

Figure 6. The main ancestry components of the different HESC lines and possible family relatedness among them. Shown are the results of the MDS based on genotypes of the HESC lines (light gray circles) together with HapMap samples (dark gray circles) from three different populations. The MDS analysis on the HapMap samples separates out three clusters corresponding to European (northern and western European ancestry; $C E U$ ), Asian (Japan and China; $J A P$ and $C H B$ ), and African (Yoruba people of Ibadan, Nigeria; $Y R I$ ) ancestry. The inset shows a separate MDS analysis on Asian HapMap individuals and CSES12. The two clusters correspond to JAP (right, light gray) and CHB (left, dark gray). CSES12 (light gray diamond) falls within the CHB cluster. 
similar to HESC lines derived from embryos that were not reported as aneuploid. These results led us to conclude that these aneuploid embryos that were due to be discarded can serve as a source for normal euploid HESC lines. In most labs, the source for derivation of HESCs comes from frozen embryos donated by families that have finished their reproductive plan. This type of embryos is rarely donated in certain populations that can afford to store the embryos for very long period. On the other hand, the majority of the couples that went through PGS donated their aneuploid embryos for research. Thus, these PGS embryos serve as an important source for deriving euploid HESCs.

We performed SNP analysis for each of the HESC lines, in order to obtain a footprint of each line and to identify their ethnic origin and family relatedness among them. Exploring the ethnic origin of the different HESC lines allows us to refine our analysis and address biological questions that are relevant to specific ethnic groups. Moreover, knowing the ethnic origin of the HESC lines will allow us to test whether there are phenotypes that are common to HESC lines from a specific ethnic origin or whether they are general for the whole population. Our HESC lines are varied, most lines fall within or very near the northern and western European cluster, one falls within the Chinese cluster, and two are a mixture between the European and the Asian clusters. Currently, we do not have HESC lines from African (Yoruba) origin. Thus we believe that there is a need to derive more HESC lines that will represent more populations.

Two pairs of HESC lines had high levels of identity by descent, suggesting that they are derived from the same parents ("full sibs"). The sibling HESC lines are a valuable resource for further investigating the effects of genetic background on the growth characteristics, pluripotency, and differentiation potential of the HESCs (Chen et al. 2009).

Acknowledgments This work was partially supported by CIRM grant RL1-00636-1: "Generation of Pluripotent Cell Lines from Human Embryos." We gratefully acknowledge support for this project provided by a grant from the Legacy Heritage Fund of New York.

Open Access This article is distributed under the terms of the Creative Commons Attribution Noncommercial License which permits any noncommercial use, distribution, and reproduction in any medium, provided the original author(s) and source are credited.

\section{References}

Adewumi O.; Aflatoonian B.; Ahrlund-Richter L.; Amit M.; Andrews P. W.; Beighton G.; Bello P. A. et al. Characterization of human embryonic stem cell lines by the International Stem Cell Initiative. Nat. Biotechnol. 25(7): 803-816; 2007.
Ao A.; Ray P.; Harper J.; Lesko J.; Paraschos T.; Atkinson G.; Soussis I. et al. Clinical experience with preimplantation genetic diagnosis of cystic fibrosis (delta F508). Prenat. Diagn. 16(2): 137-142; 1996.

Chen A. E.; Egli D.; Niakan K.; Deng J.; Akutsu H.; Yamaki M.; Cowan C. et al. Optimal timing of inner cell mass isolation increases the efficiency of human embryonic stem cell derivation and allows generation of sibling cell lines. Cell Stem Cell 4(2): 103-106; 2009.

Eiges R.; Urbach A.; Malcov M.; Frumkin T.; Schwartz T.; Amit A.; Yaron $\mathrm{Y}$. et al. Developmental study of fragile $\mathrm{X}$ syndrome using human embryonic stem cells derived from preimplantation genetically diagnosed embryos. Cell Stem Cell 1(5): 568$577 ; 2007$.

Griffin D. K.; Handyside A. H.; Penketh R. J.; Winston R. M.; Delhanty J. D. Fluorescent in-situ hybridization to interphase nuclei of human preimplantation embryos with $\mathrm{X}$ and $\mathrm{Y}$ chromosome specific probes. Hum. Reprod. 6(1): 101-105; 1991.

Itskovitz-Eldor J.; Schuldiner M.; Karsenti D.; Eden A.; Yanuka O.; Amit M.; Soreq H. et al. Differentiation of human embryonic stem cells into embryoid bodies compromising the three embryonic germ layers. Mol. Med. 6(2): 88-95; 2000.

Klimanskaya I.; Chung Y.; Becker S.; Lu S. J.; Lanza R. Derivation of human embryonic stem cells from single blastomeres. Nat. Protoc. 2(8): 1963-1972; 2007.

Lavon N.; Narwani K.; Golan-Lev T.; Buehler N.; Hill D.; Benvenisty N. Derivation of euploid human embryonic stem cells from aneuploid embryos. Stem Cells 26(7): 1874-1882; 2008.

Mitalipova M.; Calhoun J.; Shin S.; Wininger D.; Schulz T.; Noggle S.; Venable A. et al. Human embryonic stem cell lines derived from discarded embryos. Stem Cells 21(5): 521-526; 2003.

Pickering S. J.; Minger S. L.; Patel M.; Taylor H.; Black C.; Burns C. J.; Ekonomou A. et al. Generation of a human embryonic stem cell line encoding the cystic fibrosis mutation deltaF508, using preimplantation genetic diagnosis. Reprod. Biomed. Online 10 (3): 390-397; 2005.

Price A. L.; Butler J.; Patterson N.; Capelli C.; Pascali V. L.; Scarnicci F.; Ruiz-Linares A. et al. Discerning the ancestry of European Americans in genetic association studies. PLoS Genet. 4(1): e236; 2008.

Purcell S.; Neale B.; Todd-Brown K.; Thomas L.; Ferreira M. A.; Bender D.; Maller J. et al. PLINK: a tool set for whole-genome association and population-based linkage analyses. Am. J. Hum. Genet. 81(3): 559-575; 2007.

Reubinoff B. E.; Pera M. F.; Fong C. Y.; Trounson A.; Bongso A. Embryonic stem cell lines from human blastocysts: somatic differentiation in vitro. Nat. Biotechnol. 18(4): 399-404; 2000.

Robertson E. J. Embryo-derived stem cell lines. In: Robertson E. J. (ed) Teratocarcinomas and embryonic stem cells: a practical approach. IRL, Washington, pp 71-112; 1987.

Schuldiner M.; Yanuka O.; Itskovitz-Eldor J.; Melton D. A.; Benvenisty N. Effects of eight growth factors on the differentiation of cells derived from human embryonic stem cells. Proc. Natl Acad. Sci. USA 97(21): 11307-11312; 2000.

Strelchenko N.; Verlinsky O.; Kukharenko V.; Verlinsky Y. Moruladerived human embryonic stem cells. Reprod. Biomed. Online 9(6): 623-629; 2004.

Thomson J. A.; Itskovitz-Eldor J.; Shapiro S. S.; Waknitz M. A.; Swiergiel J. J.; Marshall V. S.; Jones J. M. Embryonic stem cell lines derived from human blastocysts. Science 282(5391): 11451147; 1998.

Verlinsky Y.; Strelchenko N.; Kukharenko V.; Rechitsky S.; Verlinsky O.; Galat V.; Kuliev A. Human embryonic stem cell lines with genetic disorders. Reprod. Biomed. Online 10(1): 105-110; 2005. 